\title{
Introduction to the Special Issue on Morphological Computation
}

Hauser, Helmut ; Sumioka, Hidenobu ; Füchslin, Rudolf Marcel ; Pfeifer, Rolf

DOI: https://doi.org/10.1162/ARTL_e_00083

Posted at the Zurich Open Repository and Archive, University of Zurich ZORA URL: https://doi.org/10.5167/uzh-72787

Journal Article

Published Version

Originally published at:

Hauser, Helmut; Sumioka, Hidenobu; Füchslin, Rudolf Marcel; Pfeifer, Rolf (2013). Introduction to the Special Issue on Morphological Computation. Artificial Life, 19(1):1-18.

DOI: https://doi.org/10.1162/ARTL_e_00083 


\section{Introduction to the Special Issue on Morphological Computation}

\author{
Helmut Hauser*,** \\ University of Zurich
}

Hidenobu Sumioka ${ }^{\dagger}$

Advanced Telecommunications

Research Institute International

Rudolf M. Füchslin ${ }^{\ddagger}$

Zurich University of Applied Sciences

Rolf Pfeifer**

University of Zurich

\section{Discussion}

The purposeful action of any agent in a complex environment requires control, that is, the determination of specific sequences of values for the parameters determining the state of the agent. In recent years, it became clear that we have to extend our notion of control if we want to understand the mechanical and chemical process management of biological systems. As it turns out, the lessons we learn from this extension can be directly used in engineering-foremost in the field of robotics, but increasingly also in other areas, such as artificial life or novel types of chemical systems design. The extension we refer to includes the intrinsic dynamics of the system to be controlled as an active, even computational element of control. The employment of the physical or chemical dynamics of a system as part of the computations necessary for control is the underlying principle of the concept of morphological computation. In our use of the term "morphology" we include all aspects of a physical system, not only the shape of the agent, the geometrical combination of the body parts, but also its material properties, such as friction coefficients or parameters describing compliance. Moreover, we also consider the distribution of sensors and actuators as part of the morphology.

Conventionally (and with slight oversimplification), control is understood in terms of an agent (typically the term plant is used) and a separate entity, which is the control unit and consists of a conventional digital computer. The state space of the agent is spanned by a number of parameters, which in the case of robots are mechanical in nature, but in the case of chemical cells can be densities, phase parameters, and so on. The controller possesses, via its sensors, an (at least partial) internal representation of the state of the agent at any time. In addition, the controller can change the state of the agent via some actuators. In an ideal situation, the controller exerts complete control over the agent, which means it can determine the agent's position in state space with high accuracy. In such an ideal situation, the observable behavior is the result of a conventional computation based on the internal representation of the agent in the controller. However, there is always some noise present in the dynamics of the agent. Moreover, if the model of the agent gets too complex (high-dimensional state space, high nonlinearity, noise, etc.), the required controller may be intractable. To avoid both

\footnotetext{
* Contact author.

** Artificial Intelligence Laboratory, Department of Informatics, University of Zurich, Andreasstrasse 15, 8050 Zurich, Switzerland. E-mail: hhauser@ifi.uzh.ch

$\dagger$ Hiroshi Ishiguro Laboratory, Advanced Telecommunications Research Institute International, 2-2-2 Hikaridai, Keihanna Science City, Kyoto 619-0288, Japan.

$\ddagger$ ZHAW Zurich University of Applied Sciences, Center for Applied Mathematics and Physics ZAMP, 840I Winterthur, Switzerland.
} 
problems, that is, to keep the agent controllable, an often followed strategy (during the design process) consists in the reduction of the number of degrees of freedom of the agent. For example, in the case of robots this is reflected by the use of rigid body parts, which are connected by hightorque servos.

Control based on morphological computation pursues a different strategy. There is still a division into agent and controller. However, the controller is no longer assumed to exert complete control, and therefore the line of separation gets fuzzy. In extreme cases (e.g., mechanically driven walking machines like passive walkers [1]) this separation can even vanish completely. Morphological computation requires that the physical (chemical) dynamics of the agent be designed in such a way that the complexity of the task that is solved by the controller is minimized. This happens by outsourcing parts of the computation to the physical body (more on the precise meaning of outsourcing later). In a simple example the body dynamics of the agent provide various limit cycles, and the task of the controller is reduced to choosing the right basin of attraction and initiating jumps into it. The choice will clearly depend on the demands made by the environment and/or the prescribed task. For example, in the case of a locomoting robot the dynamics of the body implicitly take over tasks such as the stabilization of the movement pattern against small perturbations, while the controller simply has to choose an appropriate gait. Stated differently, the number of tasks to be fulfilled by the conventional controller is reduced. This reduction can be understood as an outsourcing of computation to the body, since the control problem as such (e.g., how to handle small perturbations caused by an uneven floor in the case of walking machines) is still the same. Notably, not only can a clever exploitation of the physical dynamics of an agent reduce the amount of computation to be performed by the controller, but also the information necessary for the internal representation of the agent's state is reduced. As soon as the stabilization of a movement pattern is intrinsic to the body dynamics of the moving agent, the controller only needs to know in which basin of attraction the agent actually resides. No details about positions, velocities, and so on are necessary. In consequence, the computations that remain to the controller are not necessarily full simulations of the dynamics of the agent, but relatively simple evaluations such as "If condition A is satisfied and agent resides in basin of attraction $\mathrm{B}$, jump into basin C"- $\mathrm{a}$ task that is mastered by a simple finite state automaton.

It should be emphasized that morphological computation is more than clever systems design. Our claim that computation can be outsourced to the body and not just omitted is corroborated by a recent result of Hauser et al. [3, 4]. They demonstrated that a sufficiently complex physical body equipped with a simple readout (e.g., a feed-forward neural network or even only a linear and static readout) is computationally equivalent to a conventional device almost as powerful as a Turing machine. This finding has two major implications. The first one is that computation can be outsourced to the body in a quite literal sense. The second one is relevant for the engineer's choice of the design of an agent. There is a whole spectrum of options for distributing computations between the physical dynamics of the agent and a conventional control device. It is quite plausible that the optimal choice will not be found at either end of this spectrum. As briefly mentioned in the introduction, the agents under consideration need not be classical, macroscopic robots. It is well known that chemical process management in eukariotic cells results from an interplay between molecular, supramolecular, and mesoscopic structures (e.g., membranes or the cytoskeleton) and their dynamic interaction. For an illustrative example, consider protein sorting by vesicular transport [5].

Recently, simple forms of cognition and motility have been implemented in artificial systems in the laboratory; see [2]. Such systems, besides being of fundamental interest for questions concerning the origin of life as well as modern bionanoengineering, also shed light on a form of morphological control not directly visible in the macroscopic world. In the study of their dynamics, classical mechanics is replaced, or at least complemented, by statistical physics. Furthermore, collective phenomena, such as phase transitions, start to play an important role.

Morphological computation can be found on a wide range of different scales in a number of different contexts. Consequently, it has attracted many scientists from various backgrounds. For example, roboticists are interested in adopting this concept to build more energy-efficient and robust robots. Biologists try to understand the functions of body parts from the viewpoint of morphology (i.e., 
morphofunctionality). Computational scientists aim to capture the implications a physical implementation of certain types of computation has for computational theory as a whole. Scientists in the field of medicine have been attracted by the idea of morphological computation. For example, the decrease of movement control in elderly patients can, from the viewpoint of morphological computation, be interpreted as the loss of (morpho)computational power and is not necessarily the consequence of decreasing neural activity. The cause of this loss can be found in a change of the mechanical properties of the body - a change that also alters the attractor landscape in the state space of the body. As a result of aging, this attractor landscape may become less pronounced (e.g., self-stabilization of movement patterns may become slower) and be more difficult to navigate.

The interdisciplinarity of the research community suggests that morphological computation is a very general concept. On one hand, this represents a significant challenge when we try to capture the concept in a unified theory or even if we want to define the boundaries of the field itself. On the other hand, it provides us with numerous research opportunities, especially in the interdisciplinary, overlap areas of research. We believe morphological computation even has the potential to give rise to completely new fields of research. To harvest this inherent potential of our community, we organized the 2nd International Conference on Morphological Computation (http://morphcomp.org/) in September, 2011, in Venice. As one of the results of the conference we initiated this special issue. The conference committee and all invited speakers, all experts in the field, were asked to vote for their favorite submissions among the accepted abstracts. Based on this poll, we invited the corresponding authors to submit an extended journal version of their work.

As a result, we have in total nine accepted articles, which provide us with a snapshot of recent research done in the context of morphological computation. Furthermore, they also point to new directions of research in the field. While some of the articles have a focus on engineering (e.g., building a series of real robots to investigate the influence of morphology on locomotion, as in Reis et al.'s article), others are oriented toward more general considerations and point to potential future research directions. One from the latter category is the submission by Herrera and Sanz. Their article illustrates impressively how morphological computation attracts an interdisciplinary audience. The background of the authors is found in the theory of emotion. They suggest linking emotions with changes in the morphology and the resulting changed functionality. For example, if the emotion of fear arises, the body changes, namely, it gets ready- either to fight or to flee. As a consequence, the body can no longer be described as a fixed dynamical system, but has to been seen as a variable dynamical structure, which changes its state of attraction (from a finite and discrete set) depending on the emotion it embodies at the moment. This approach implies a new way of looking at the embodiment in robotics and constitutes a new challenge for the design and control of such physical bodies. Although such emotion-induced changes will take place on a slower timescale than the usual movements, the control is nontrivial. The controller will have to produce discrete switches between analogue systems in a stable fashion. The authors suggest some potential control theoretical approaches; however, we might have to rethink the control approach for such systems entirely.

Another article, which also belongs to the more general category of this special issue, is the one by Füchslin et al. It consists of two parts: First, it presents and discusses a formalization of the concept of morphological computation. The authors build upon the Venice definition ${ }^{1}$ given at the First International Conference on Morphological Computation. They clarify the notions of result and input, and the notion and role of a program in morphological computation. Such a formalization is helpful when one aims at a better understanding of what it means to program a morphological computation. It is often illustrated using attractor landscapes of dynamical systems. Füchslin et al. discuss also what it means if the controller (the brain) can alter the attractor landscape. They interpret, for example, training in various sport activities not as learning how to move, but learning how to bring the body into a morphology (internal tension, geometry) that is optimally suited for a given task. Based on different types of feedback between agent and controller (body and brain), they present a hierarchy

\footnotetext{
I Morphological computation has informally been defined as any process that (a) serves a computational purpose, (b) has clearly assignable input and output states, and (c) is programmable.
} 
of levels of morphological computation. In a second part, they present three partially ongoing case studies of systems employing morphological computation and control in different contexts. A first case study deals with exoskeletal support systems for people with movement impairments. In a second investigation, they analyze self-assembly processes from the viewpoint of morphological computation. The third study is more speculative and discusses aspects of cellular dynamics in a specific type of combination therapy in oncology with respect to control.

Umedachi et al. investigated morphological computation on the level of single-cell organismsspecifically, the true slime mold. This is a large amoeba-like unicellular organism that does not have any nervous system. Nevertheless, it exhibits a surprisingly rich set of behaviors. In order to understand how such complex patterns can emerge without any high-level cognitive abilities (or even any simple neural networks), they implemented a simple physical model. In their simulations they induce changes of behavior (such as taxiing, exploratory, and escape behaviors) through physically interacting mechanosensory oscillators, solely by mechanically stimulating the agent, without changing the parameters of the model itself. This clearly points to the importance of the physical (morphological) layer, which allows the emergence of complex behavior. Further investigation in that direction can shed light on how biological systems can exhibit behavioral diversity based on a clever morphology. Understanding the underlying concepts will allow us to build lifelike robots with the capability of having versatile and adaptive behavior already encoded in the physical layer. This is especially interesting for highly decentralized systems, that is, systems where no global controller is present to decide what to do next.

Although the concept of morphological computation implies that a computation can be taken over, at least in part, by the physical body of an agent, it is nontrivial to quantify to what extent this happens. How much of a computational task can be (or should be) outsourced, and how much is therefore left for the high-level controller? These questions have been investigated by Rueckert and Neumann. In their setup they simulated a simple four-link model of a humanoid robot in twodimensional space. They applied random forces to the robot and taught an optimal controller by stochastic learning techniques to counteract these perturbations. They defined a measure to capture the "complexity" of the found controllers, and thereby indirectly identified how much of the computational task had been outsourced to the physical body. They investigated different parameters, like friction and lengths of different body parts, and their influence on the shift of computation from the controller to the physical part of the robot. They even added compliance to the body and looked for optimal spring constants. Their results clearly demonstrate how computation can be transferred to the body, resulting in simpler controllers at a higher level. While their measure of complexity is closely related to their setup (i.e., to their applied learning technique), their results point to a research opportunity to find more general methods to quantify morphological computation.

The idea of morphological computation implies that the design process for robots will have to be revised significantly. Properties typically undesired in today's robot design, such as compliance, ${ }^{2}$ nonlinearities, and high dimensionality, are now to be considered beneficial for a computationally powerful robot (see Hauser et al. [3, 4]). As a consequence, new types of design approaches and eventually a novel class of robots will arise. A step in that direction has been made by Caluwaerts et al. in this issue. Instead of using classical shapes for their robots (i.e., bio-inspired forms) they investigated tensegrity structures. Their choice was based on the properties of these structures and not made because they resemble any specific animal or because they fit a given task best. This is a new way of looking at robots induced by morphological computation. The authors demonstrate how such complex, compliant physical systems can locomote when stimulated only by very simple controllers. Since the complex coordination is done within the physical domain, the resulting controller on top of it can be simple to the extent of being linear and static. As a consequence, the controller parameters can be easily learned online. This points to the fact that morphology not only allows for simpler controllers,

2 The editors are aware of the increasing interest in compliant actuators and their applications in robots. However, we suggest that an even more radical implementation of compliance in the robot's body should be considered: soft skin, new types of material to build the support structure, and the like. 
but also simplifies the corresponding learning task. This might be an explanation of the fact that biological systems, despite their complex bodies, are highly adaptive and robust.

Simulations are a useful tool to investigate different possible morphologies. However, there are limitations, as it is not possible to catch every single aspect of the real world. Therefore, a reasonable approach to understand underlying principles of morphological computation is to build robots with different morphologies and investigate them systematically. For example, Reis et al. built a series of robots with specific differences in their morphology. This allowed them to investigate the influence of certain morphological characteristics in a systematic way and to compare the results with their mathematical model. They demonstrated that quite a simple structure can exhibit various behaviors (in their case different gaits), and how different morphologies can be beneficial.

As pointed out earlier, by morphology we not only refer to the shape, but also include material properties, positions of sensors and actuators, friction, and so on. Dermitzakis et al. concentrated in their work on one aspect, namely friction in the case of grasping. Biological data suggest that friction plays an important role, if properly employed. The authors propose a simple model and compare their data with data on real hands. Note that friction is typically avoided in robot hand design, as it is usually regarded as a cause of energy loss. However, morphological computation offers an alternative point of view and suggests that, if the design has been made cleverly enough, friction can be of advantage.

Miyashita et al. investigated morphological computation in the context of self-assembly. Although they do not implement robots on the molecular level, they investigate how morphology under such low-scale conditions (noise, stochastic interaction, no external controller, etc.) could give rise to more complex structure and behavior. Their study deals with self-organization in which distributed centimeter-size modules stochastically aggregate and exhibit a translational wheeling motion. They demonstrate that the right morphology can give rise to self-organization in a robust fashion. Their results may shed light on nonequilibrium processes in membranes. Their macroscopic system is generically two-dimensional (it consists of flowing tiles) and may serve as an experimental platform for the study of aggregation processes as they occur in cellular process management.

In looking at the various instantiations of morphological computation that can be found in nature, it is a reasonable approach to take a closer look at the morphologies of biological systems. One approach is, for example, to build anthropomimetic robots and see how this specific morphology is of advantage. The EU project ECCE (http://eccerobot.org/) took this approach. Wittmeier et al., in this issue, summarize their results of four years of work, and also report the challenges they have faced by taking this approach. Over the years they built four versions of an anthropomimetic human torso, increasing in their complexity and number of details. They developed a sophisticated simulation of the robot and a number of control techniques. Some of the controllers are based on classical control theory, while others use machine learning techniques to deal with the complex structure. An additional approach was made by using motor primitives as a control framework. In any case, controlling such a complex structure (from the viewpoint of system theory this is a very high-dimensional and nonlinear system) is a nontrivial task. The presented results are impressive; one of the authors' main contributions was to explicitly show the opportunities for further research in the context of an anthropomimetic approach.

\section{Research Opportunities}

The community of scientists investigating morphological computation is a vibrant and fast-growing group. As this special issue demonstrates, exciting results have been obtained so far, but they also point to a number of research opportunities. Here is a noncomprehensive list of possible research directions for morphological computation.

\section{I Design Principles}

If we fully embrace the concept of morphological computation, we eventually come to an entirely new way to look at morphology. As a consequence, the physical body of a robot will have to be considered much more in the design process than it is today. We believe the change will be drastic, 
even revolutionary. Robots will not only look different, but will also be built out of new types of materials that have properties that support morphological computation. Caluwaert et al., with their use of tensegrity structures as a robotic device, have already pointed in that direction. Also, the robots built by Reis et al. exhibit a new look in that they do not target resemblance in form to any particular animal. Another contribution to this special issue, by Umedachi et al., also reminds us to reconsider classical robot design. Their intended applications can be found on the micro scale and within heavily decentralized artificial forms of life. Also with respect to prosthetics, morphological computation can inspire novel directions. In one of their case studies, Füchslin et al. discuss a support system for patients with movement impairments that aims at reestablishing controllability of the body, but does not directly guide movements. Altogether, this new generation of robots based on new emerging design principles will be in general more energy efficient, robust, and highly versatile.

\subsection{A New Type of Control: Orchestration}

An implication of these new types of robots is that the clear separation between the controller and the to-be-controlled will eventually vanish. Such a situation cannot be captured by classical control theory. However, even these new types of robots will have to be controlled. Therefore, we are going to need a new type of control, complementary to classical control theory, which we refer to as orchestration. The controller can be considered like the conductor of an orchestra. The individual "musicians" are the body parts, including their local sensing and actuation circuitry. They are acting to a certain extent autonomously and are not controlled at every single instant of time, but rather they are guided by the conductor. To give an example, in the case of a locomoting robot, the musicians would be body parts, that is, mechanical structures with their local sensors and actuators, which are able to counteract small perturbations on a local level. Mathematically they would be represented as stable limit cycles. The conductor would only have to switch between different gaits (i.e., different limit cycles) to adapt to environmental changes.

\subsection{Learning with Morphologies}

Closely related to the control problem is the task of learning to control the body. As previously pointed out, the use of a clever morphology can help to reduce the complexity of the corresponding learning task. Rueckert and Neumann showed in their contribution an approach to a simple 2D model of a humanoid. However, robots that will take radical advantage of morphological computation will typically be very high-dimensional, compliant, and nonlinear. We will have to find new frameworks to understand how learning can take place here. The learning algorithms will have to take advantage of the structure and should be able to exploit the given morphology.

Another possibility is to let the controller coevolve with the body. While this has to be done in simulations for now, we hope in the future to see materials capable of growing and to see implementations in growing robots.

\subsection{Understanding the Tradeoff Space}

If we incorporate functionality into the physical body, we gain on one hand in that we can outsource computation to the morphology, but on the other hand we lose flexibility. There is a tradeoff space we need to understand to fully exploit morphological computation principles. Extreme examples in that context are passive walkers (see, e.g., [1]). Essentially all their (control) computation takes place in the physical realm (i.e., in the mechanical structure of the robot and its interaction with the environment). While it is impressive to see such implementations, these robots completely fail when walking under different environmental conditions (different slopes, rough ground, etc.). We also have to consider that the physical properties of the material used introduce constraints. For example, there is a natural bandwidth limitation for signals to travel along compliant structures, because they act as lowpass filters. Furthermore, we will have to explore the energy efficiency of mechanical structures when they are exploited for computation. 


\subsection{Theoretical Frameworks}

Another research opportunity is to develop theoretical frameworks for morphological computation. It is desirable to have mathematical models to predict which properties are beneficial for computation. It has been only lately that there has been some progress in this context. For example, it has been shown by Hauser et al. [3, 4], by introducing two different mathematical models for morphological computation, that using compliant physical bodies can contribute to computation.

Conventional computation is defined by the concept of a Turing machine. Such a paradigm is lacking in morphological computation. Füchslin et al., in this special issue, propose a mathematical framework to define morphological computation. As they discuss, their definition captures relevant aspects of what one intuitively would regard as essential for morphological computation, but there are processes that can sensibly be regarded as instances of morphological computation but are not covered by their definitions. It may be necessary to define several independent types of morphological computation in order to keep definitions transparent. Conventional computation (to be more precise: the simulation of physical processes by conventional computation) is based on a translation of the physical reality into sequences of characters. Füchslin et al. claim that this translation offers a lot of advantages, but also carries a price. Certain rather basic processes, such as the interaction of unevenly shaped extended objects, turn out to be difficult to capture by efficient algorithms processing (binary) sequences. Even if one does not believe in a fundamental difference between conventional and morphological computation (in the end, a computer also is a physical system), theoretical considerations should clarify whether there are morphocomputational systems that one can regard as versatile and programmable and that efficiently solve problems that are computationally intensive when using conventional computers.

\subsection{Quantification of Morphological Computation}

Closely connected to the theoretical models is the question of quantification. Rueckert and Neumann were able to define a measure of complexity for their controllers that was directly linked to their approach. However, it is desirable to find more general definitions to measure the complexity of computation and the related morphology. One possible way is to employ well-established techniques from information theory. However, we believe that new types of mathematical frameworks might even be needed.

\subsection{Growing Morphologies}

As shown in Rueckert and Neumann's article, morphologies can be optimized for certain tasks. However, a real autonomous robot, which has to interact in such a dynamic and complex world as ours, has to be able to deal with a huge number of different situations. Hence, adaptivity is a key issue for autonomous robots. This means that the morphology has to be changed according to the task. We believe that real adaptation (and therefore robustness) goes beyond change of some parameters or of posture, and should include growth. Unfortunately, materials science is not (yet) ready to provide sophisticated materials that allow robots to grow. Nevertheless, it is only a question of time until we have such tools available, and we believe morphological computation will benefit from them.

\section{The Future of Morphological Computation}

Morphological computation has come a long way from the first observations of the concept in nature to where we are now. Nevertheless, there is still much to do. The boundaries of the field are not well established, a good mathematical foundation is lacking, and applications are mostly missing. However, we believe we are right now in an exciting era for morphological computation research, as one can see in this special issue. The core community of roboticists seems to be slowly embracing the concept. There is a clear trend in building compliant robots, especially with regard to safe interaction with humans. Materials science is making immense progress in new materials beneficial to 
morphological computation. Finally, the research community utilizing morphological computation is growing fast. We are looking forward to seeing more exciting results and a new generation of robots. Eventually the idea of morphological computation will be turned into a paradigm, complemented by sophisticated tools and applications.

\section{Acknowledgments}

We would like to thank all the reviewers of this special issue for their time and their helpful suggestions. We also would like to thank Mark Bedau and Kathleen Kennedy for their kind support and their patience.

\section{References}

1. Collins, S., Ruina, A., Tedrake, R., \& Wisse, M. (2005). Efficient bipedal robots based on passive-dynamic walkers. Science, 307, 1082-1085.

2. Hanczyc, M. M. (2011). Metabolism and motility in prebiotic structures. Philosophical Transactions of the Royal Society B: Biological Sciences, 366(1580), 2885-2893.

3. Hauser, H., Ijspeert, A. J., Füchslin, M. R., Pfeifer, R., \& Maass, W. (2012). Towards a theoretical foundation for morphological computation with compliant bodies. Biological Cybernetics, 105, 355-370.

4. Hauser, H., Ijspeert, A. J., Füchslin, M. R., Pfeifer, R., \& Maass, W. (2012). The role of feedback in morphological computation with compliant bodies. Biological Cybernetics, 106, 1-19.

5. Rothmann, J. E., \& Wieland, F. T. (1996). Protein sorting by transport vesicles. Science, 272, 227-234. 